\title{
Recuo assimétrico dos músculos retos horizontais para correção de incomitância alfabética: relato de caso
}

\author{
Asymmetric recession of the horizontal rectus muscles for correction alphabetical incomitance: case report
}

Alyne Borges CorrêA ${ }^{1}$, Tomás Fernando Scalamandré Mendonç̧A ${ }^{1}$

\section{RESUMO}

Os autores relatam o caso de um homem de 21 anos com estrabismo divergente incomitante, anisotropia em "V", hiperfunção de músculo oblíquo inferior direito e hipofunção de obliquo superior direito, no qual foi realizado, sob anestesia tópica um recuo assimétrico das fibras dos músculos retos horizontais para correção da incomitância alfabética. O resultado cirúrgico imediato foi considerado muito bom (ortotrópico e sem incomitância alfabética), já que pela técnica cirúrgica convencional não se obteve sucesso.

Descritores: Estrabismo; Exotropia; Estrabismo/cirurgia; Músculos oculomotores/ cirurgia; Procedimentos cirúrgicos oftalmológicos/métodos; Relatos de casos

\begin{abstract}
The authors report a case of 21-year-old man with divergent noncomitant strabismus, " $V$ " pattern anisotropy, right inferior oblique muscle overaction and right superior oblique muscle hypofunction, which was performed under topical anesthesia an asymmetrical recession of the horizontal rectus muscles fibers to correct alphabetical incomitance. The immediate surgical outcome was considered very good (orthotropic, no "V" or "A" pattern), since the success was not obtained through conventional surgical technique.
\end{abstract}

Keywords: Strabismus; exotropia; Strabismus/surgery; Oculomotor muscles/surgery: Ophthalmologic surgical procedures/methods; Case reports

\section{INTRODUÇÃO}

As anisotropias alfabéticas são formas de estrabismo em que o tamanho do ângulo de desvio horizontal varia entre supraversões e infraversões, ou seja, apresentam incomitâncias horizontais quando os olhos se movem para cima e para baixo, ao redor de um eixo frontal que passa pelo centro de rotação do olho, o eixo $X$ de Fick. Apresentam-se, principalmente nas formas de anisotropias em " $A$ " e " $V$ ". Existem outros padrões menos frequentes de anisotropias verticais: $X, Y$ e $\lambda$, que são consideradas variações dos padrões em "A" e em " $\bigvee$ "(1,2).

$\mathrm{Na}$ anisotropia em " $\mathrm{A}$ " encontramos uma esotropia (ET) maior em supraversão do que em infraversão ou exotropia (XT) maior em infraversão do que em supraversão. Ao contrário, na anisotropia em " $V$ " ocorre ET maior em infraversão do que em supraversão ou XT maior em supraversão do que em infraversão(1). Em resumo, na incomitância em " $A$ " os olhos estão mais afastados entre si em infraversão do que em supraversão e na incomitância em "V" estão mais próximos entre si em infraversão do que em supraversão.

A causa das anisotropias alfabéticas já foi atribuída às disfunções dos músculos retos horizontais ${ }^{(3)}$. Também foi descrito que o problema primário estaria nos retos verticais e secundariamente nos oblíquos ${ }^{(4)}$. Atualmente, atribui-se como causa da maioria das incomitâncias alfabéticas a disfunção dos músculos oblíquos, mas há casos em que não são observados acometimento destes músculos.

Quando existe disfunção de músculos oblíquos, as cirurgias são realizadas nestes músculos. Na anisotropia em "A" é comum a hiperfunção dos oblíquos superiores (OSs) e mais raramente hipofunção dos oblíquos inferiores (Ols). Na anisotropia em "V" é comum a hiperfunção dos Ols ou hipofunção dos OSs. A cirurgia visa enfraquecer os músculos oblíquos hiperfuncionantes, ou reforçar os hipofuncionantes.

Quando ocorre anisotropia sem disfunção dos músculos oblíquos, a técnica cirúrgica utilizada com mais freqüência é o deslocamento vertical dos retos horizontais ${ }^{(5,6)}$, pois isto modifica o seu vetor de força conforme o olho se movimenta. Por exemplo: quando o eixo medial é deslocado inferiormente, sua força fica menor em infraversão do que em supraversão, por isso corrige incomitância em " $V$ ". Outras técnicas que podem ser utilizadas são: deslocamento dos retos verticais medialmente ou lateralmente; e o deslocamento vertical unilateral dos retos horizontais. Para corrigir o padrão em "A" é realizado o deslocamento superior dos músculos RMs e/ou deslocamento inferior dos RLs. Quanto ao padrão em "V", a correção é realizada com o deslocamento inferior dos RMs ou superior dos $R L s^{(1)}$. No deslocamento horizontal dos retos verticais, altera-se a ação adutora destes músculos, que é aumentada se o deslocamento for medial e reduzida se for temporal. Desta maneira, os retos superiores (RSs) podem ser deslocados temporalmente quando existe " $A$ " $\mathrm{e}$ medialmente quando " $\vee$ ", enquanto os retos inferiores (RIs) são deslocados temporalmente em " $V$ " e medialmente nas incomitâncias em "A"(1). O deslocamento vertical de ambos retos horizontais do mesmo olho foi proposto por Goldstein ${ }^{(7)}$, no entanto, tal procedimento pode causar torção ocular.

Bietti em 1970(8) propôs uma maneira de corrigir as incomitâncias alfabéticas através do recuo assimétrico das fibras dos músculos retos
Submetido para publicação: 24 de novembro de 2011

Aceito para publicação: 22 de junho de 2013

Trabalho realizado no Departamento de Oftalmologia da Universidade Federal de São Paulo UNIFESP - São Paulo (SP) Brasil.

Médico, Departamento de Oftalmologia, Escola Paulista de Medicina, Universidade Federal de São Paulo - UNIFESP - São Paulo (SP), Brasil.
Financiamento: Não houve financiamento para este trabalho.

Divulgação de potenciais conflitos de interesse: A.B.Corrêa, Nenhum; T.F.S.Mendonça, Nenhum. Endereço para correspondência: Tomás Fernando Scalamandré Mendonça. Rua Botucatu, 821 São Paulo (SP) - 04023-062 - Brasil - E-mail: alynegbcorrea@hotmail.com

Comitê de Ética: Aprovado no CEP UNIFESP, número: 1476/10 
horizontais. Em seu estudo, para os casos de ET com " $V$ ", recuou mais a parte inferior dos RMs, nos casos de ET com " $\mathrm{A}$ " recuou mais a parte superior dos RMs. Para os pacientes com XT e " $V$ " foi recuado mais a parte superior dos RLs e nos casos de XT com "A" o recuo dos RLs foi maior na parte inferior. A diferença entre a parte superior e a inferior dos músculos recuados foi de $2 \mathrm{~mm}$ em cada caso.

O método utilizado foi um recuo assimétrico das fibras dos músculos retos horizontais, seguindo o mesmo raciocínio de Bietti, porém de uma maneira modificada, onde a assimetria não fica limitada pela largura do músculo. Inicialmente é feito um "split" (divisão ao meio das fibras musculares superiores e inferiores) e a seguir, em cada hemimúsculo é feita uma sutura tipo "hang back". Assim, é possível recuar cada metade do músculo de maneira bem assimétrica e na quantidade necessária para correção da incomitância.

\section{RELATO DE CASO}

Paciente do sexo masculino, 21 anos, com história de desvio ocular desde a infância que piorou na adolescência.

Ao exame apresentava refração estática no olho direito (OD) de $-0,25$ DE -0,75 DC $120^{\circ}$ (acuidade visual 1,00) e no olho esquerdo (OE) -0,50 DE -0,50 DC $30^{\circ}$ (acuidade visual 1,00). Ao exame de motilidade ocular, XT de $40^{\Delta} \mathrm{com}$ hipertropia à direita (HTD) de $5^{\Delta} \mathrm{em}$ posição primária do olhar (PPO) e anisotropia em " $V^{\prime \prime}$ de $25^{\Delta}$ (XT de $50^{\Delta}$ em supraversão e XT de $25^{\Delta}$ em infraversão). Nas versões: -1 de reto lateral direito (RLD), +4 de oblíquo inferior direito (OID) e -2 de oblíquo superior direito (OSD). Em destroversão XT de $35^{\Delta}$ e HTD $2^{\Delta}$ e em levoversão XT de $40^{\Delta}$ e HTD $30^{\Delta}$ (Figura 1). Ao teste de Maddox, extorsão de $5^{\circ}$ no OD. Biomicroscopia, pressão intraocular e fundo de olho sem alterações.

Foi indicada cirurgia corretora de estrabismo sob anestesia tópica (apenas colírio anestésico e leve sedação) para ajustes per-operatórios. Todas as avaliações per-operatórias do desvio foram realizadas com o paciente sentado fixando objetos tanto para longe quanto para perto, nas diferentes posições do olhar.

Realizamos em um primeiro momento um recuo do RLD de 8 milímetros $(\mathrm{mm})$ a partir de sua inserção e miectomia do OID. Porém, apesar de eliminada a hiperfunção do OID, persistia tanto o XT quanto a anisotropia em " $V$ ". Então um recuo do RLE de $8 \mathrm{~mm}$ foi efetuado, com elevação de uma inserção, ou seja, a extremidade inferior do RLE foi reposicionada $8 \mathrm{~mm}$ mais distante da sua inserção original e na altura de onde antes era a sua extremidade superior. Apesar de terem diminuído, tanto o XT quanto a anisotropia em "V" permaneceram. A seguir, o músculo RMD foi ressecado $4 \mathrm{~mm}$ e a nova inserção foi deslocada $3 / 4$ (aproximadamente $7 \mathrm{~mm}$ ) inferiormente. Neste momento, o XT estava corrigido em PPO, porém, ainda persistia a incomitância em $\mathrm{V}$.
Na tentativa de correção da incomitância em " $\vee$ " remanescente, optamos por modificar a forma de recuo que havia sido realizada no RLE, da seguinte maneira: o músculo foi dividido longitudinalmente ao meio, até $15 \mathrm{~mm}$ posterior à sua inserção prévia, sendo a parte superior do RLE recuado $12 \mathrm{~mm}$ e a parte inferior mantida a $8 \mathrm{~mm}$, a partir da inserção. Foi observada uma melhora importante do $\mathrm{V}$, persistindo, porém desvio residual.

Em seguida, optamos por realizar o mesmo procedimento no RLD. O músculo foi dividido ao meio, até $15 \mathrm{~mm}$ posterior à sua inserção prévia, a parte inferior foi mantida recuada a $8 \mathrm{~mm}$ e a parte superior recuada $12 \mathrm{~mm}$ de sua inserção (Figura 2).

Observamos no per-operatório que o paciente ficou ortotrópico em PPO, sem hipertropia e a anisotropia em $\vee$ foi resolvida.

No sétimo dia de pós-operatório o paciente permanecia ortotrópico, sem extorsão ao Maddox e sem incomitância alfabética.

\section{DISCUSSÃO}

O recuo em "leque" das fibras dos retos horizontais para correção da incomitância alfabética proposto por Bietti ${ }^{(8)}$ tem suas limitações pela própria anatomia do músculo, ou seja, a assimetria do procedimento é limitada pela largura do músculo, o que não ocorre com o método que aqui propomos, sendo possível recuar cada metade do músculo na quantidade necessária para correção da incomitância.

A vantagem em relação à transposição vertical dos músculos horizontais é que não há indução do desvio vertical e se mantém a linha de ação do músculo(7,8).

Neste caso, onde já havíamos feito uma miectomia do Ol hiperfuncionante, deslocamento das inserções dos músculos RLE e RMD, e ainda assim a incomitância alfabética persistia, optamos por este procedimento de recuo assimétrico dos retos laterais com uma assimetria maior do que a proposta por Bietti, e conseguimos um resultado cirúrgico satisfatório. Ou seja, apesar da técnica descrita ter sido realizada em um músculo com inserção elevada anteriormente, ela somente foi feita após a avaliação per-operatória de que a elevação da inserção não teria sido suficiente para a correção total da incomitância em V.

A realização desse procedimento sob anestesia tópica foi de importância fundamental neste caso, pois pudemos observar no peroperatório que, mesmo após a realização dos procedimentos convencionais, a incomitância em " $V$ " persistia e melhorou nitidamente após o recuo assimétrico dos músculos retos. O grau de vigília do paciente, bem como avaliação no momento da cirurgia, nos permitiu uma avaliação eficaz no per operatório, pois foi realizado com mínima sedação, apenas colírio anestésico como anestesia e solicitando que o paciente ficasse sentado e fixando objetos tanto para perto quanto para longe, nas diferentes posições do olhar.
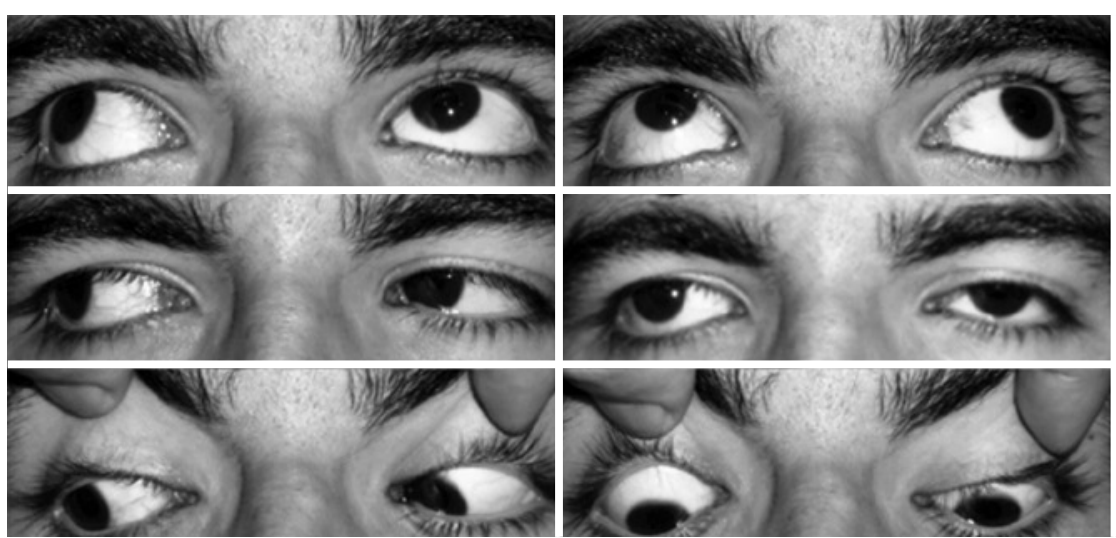
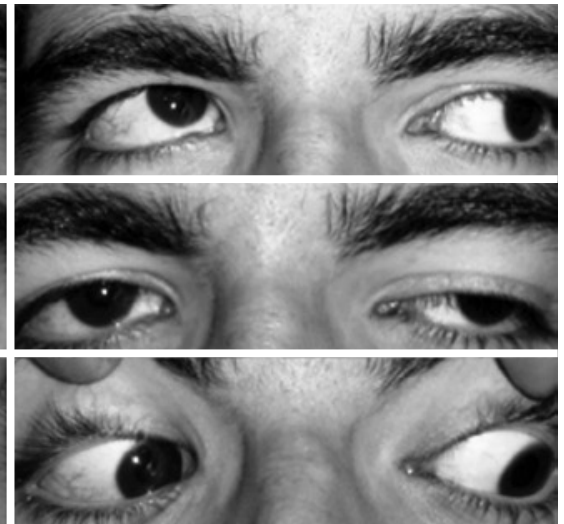

Figura 1. Versões. 


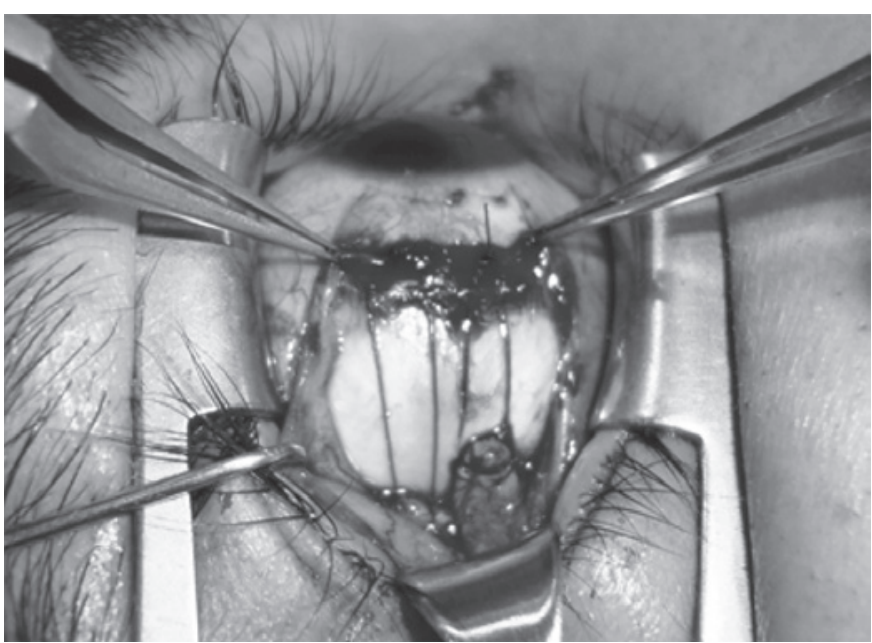

Figura 2. Recuo assimétrico do músculo reto lateral direito (RLD).

Conforme observado no momento da cirurgia, somente a miectomia e a transposição não foram suficientes para a correção do " $V$ " neste caso especificamente, e acreditamos que a realização dessas alterações em três tempos provavelmente teriam os mesmos resultados, porém, gerariam maiores despesas e transtornos ao paciente.
Um novo estudo, do tipo caso/controle, já está em andamento, utilizando apenas a técnica modificada de recuo assimétrico dos músculos retos horizontais, sem associação de miectomia do Ol e sem deslocamento vertical das inserções, para podermos padronizar melhor sua eficácia.

O método que aqui descrevemos é uma alternativa de correção das incomitâncias alfabéticas para os casos sem disfunção de músculos oblíquos, ou mesmo nos casos em que a cirurgia realizada nestes músculos não for suficiente para corrigir a incomitância alfabética. Este novo procedimento provavelmente causa menor efeito torsional, pois não há deslocamento de inserções.

\section{REFERÊNCIAS}

1. Prieto-Díaz J, Souza-Dias C. Estrabismo. 2a ed. São Paulo: Roca;1986. p.247-85.

2. Wright KW. Strabismus and pediatric ophthalmology. New York: Springer-Verlag; 2003. p.297-309.

3. Urist M. Recession and upward displacement of the medial rectus muscles in A pattern esotropia. Am J Ophthalmol. 1968;65(5):769-73.

4. Brown HW. Symposium strabism, vertical deviations. Trans Am Acad Ophthalmol Otolaryngol. 1953;57(2):157-62.

5. Knapp P. Vertically incomitant horizontal strabismus. The so-called " $\mathrm{A}$ "' and " $\vee$ " syndromes. Trans Am Ophthalmol Soc 1959;57:666-99.

6. Von Noorden GK, Burian HM. Binocular vision and ocular motility. St. Louis, The C.V. Mosby Co 1974; p.337-40

7. Goldstein JH. Monocular vertical displacement of the horizontal rectus muscles in the $A$ and $V$ patterns. Am J Ophthalmol. 1967;64(2):265-7.

8. Bietti GB. [On a technical procedure (recession and fan-shaped oblique reinsertion of the horizontal rectus muscles) for correction of $\mathrm{V}$ or $\mathrm{A}$ exotropias of slight degree in concomitant strabismus]. Boll Ocul. 1970;49(11):581-8. Italian. 